\title{
Radial plant growth - Cellular coordination during growth in two dimensions
}

\author{
Shi Dongbo, Wallner E.-S., Brackmann K., Qi Jiyan, Schlamp T., \\ Chiang Min-Hao, Greb T.* \\ Centre for Organismal Studies (COS), Heidelberg University, Heidelberg, Germany \\ *e-mail: thomas.greb@cos.uni-heidelberg.de
}

Body shaping in multicellular organisms depends on the activity of distinct stem cell niches coordinated over long distances. Radial growth of plant shoots and roots is a stem cell-driven process fundamental for the mechanical and physiological support of enlarging plant bodies. In most dicotyledonous species, the underlying stem cell niche, the cambium, displays a strictly bifacial character generating xylem (wood) inwards and phloem (bast) outwards. Despite its importance and intriguing dynamics, the functional characterization of cambium stem cells was hampered by the lack of experimental tools for accessing distinct cambium sub-domains. Investigating the hypocotyl of Arabidopsis thaliana, we mapped stem cell activity in the proliferating cambium. Through pulselabelling and genetically encoded lineage tracing, we established different transgenic markers defining a proximal, a central and a distal cambium domain. We further demonstrated that the proximal domain represents a site of xylem formation and the distal cambium domain contains cells determined for phloem development. Moreover, using tissue-specific transcriptomics, chromatin analysis and local genetic perturbation, we unravelled regulatory circuits specifically regulating the transition of common stem cells to xylem or phloem tissues. Thereby, our analysis provides a cellular fate map of a strictly organized plant meristem and reveals determinants instructive for a bifacial tissue production. 